\title{
Aporte e Decomposição da Serapilheira na Caatinga no Sul do Piauí
}

\author{
Rissele Paraguai Lima ${ }^{1}$, Milton Marques Fernandes ${ }^{2}$, Marcia Rodrigues de \\ Moura Fernandes ${ }^{3}$, Eraldo Aparecido Trondoli Matricardi ${ }^{3}$ \\ ${ }^{1}$ Curso de Engenharia Florestal, Universidade Federal do Piauí - UFPI, Bom Jesus/PI, Brasil \\ ${ }^{2}$ Departamento de Ciências Florestais, Universidade Federal de Sergipe - UFS, Aracaju/SE, Brasil \\ ${ }^{3}$ Departamento de Engenharia Florestal, Universidade de Brasília - UnB, Brasília, DF, Brasil
}

\begin{abstract}
RESUMO
Estudos de ciclagem de nutrientes no bioma Caatinga podem contribuir para o melhor entendimento dos processos ecológicos nesse ecossistema. Dessa forma, o presente estudo buscou ampliar as informações sobre o aporte do material formador da serapilheira, sua composição química e velocidade de decomposição em uma área de Caatinga no município de Santa Luz, PI. A produção de serapilheira foi coletada mensalmente (dezembro de 2010 a novembro de 2011), utilizando-se 10 coletores. Parte do material coletado foi utilizada para análise de N, K, P, lignina e tanino. A decomposição foi avaliada utilizando litter bags. Os resultados deste estudo indicam que a redução da precipitação levou ao aumento da deposição de serapilheira, principalmente a fração folhas. O nitrogênio apresentou o maior aporte dentre os nutrientes. Os elevados teores de lignina e tanino na serapilheira da Caatinga reduzem a velocidade da decomposição da serapilheira.
\end{abstract}

Palavras-chave: ciclagem de nutrientes, floresta tropical seca, material decíduo.

\section{Litter Contribution and Decomposition in the Caatinga in Southern Piauí State, Brazil}

\begin{abstract}
Studies on nutrient cycling in the Caatinga biome can support a better understanding of the ecological processes in that ecosystem. This study aimed to improve information related to the amount of organic material for litter formation, its chemical composition and required time for decomposition in a study area located in the Caatinga biome, municipality of Santa Luz, Piaui state, Brazil. The litterfall production was monthly collected between December 2010 and November 2011 using 10 collectors. The collected material was partially used as samples for soil analysis (N, K, P, lignin and tannin). The decomposition was assessed using litter bags. Our results indicate that litter deposition increases as precipitation decreases, mainly the leaf fraction. The greatest soil nutrient content was Nitrogen. It seems that the high concentrations of lignin and tannin in the Caatinga litter were responsible for decreasing the decomposition rate of the leaf litter.
\end{abstract}

Keywords: nutrient cycling, dry tropical forest, material deciduous. 


\section{INTRODUÇÃO}

Por ser um fator chave na manutenção dos nutrientes no ecossistema, o processo de deposição da serapilheira, incluindo as taxas anuais de queda do material decíduo e o processo de decomposição desse material, devem ser mais amplamente estudados e conhecidos, especialmente nas condições dos trópicos, onde há grande ocorrência de solos com baixos níveis de nutrientes (Santana \& Souto, 2011).

Para Pinto et al. (2008), são vários os fatores bióticos e abióticos que comprometem a produção de serapilheira, como tipo de vegetação, altitude, latitude, precipitação, temperatura, regimes de luminosidade, deciduidade da vegetação, estádio sucessional, disponibilidade hídrica e características do solo.

A morfologia, fisiologia e ecologia das plantas da Caatinga determinam as características vegetacionais do bioma. Assim, os processos biológicos, dado o comando genético, selecionaram peculiaridades adaptativas a altas temperaturas e baixa precipitação, tornando a flora endêmica da Caatinga compatível com as condições severas a que estão sujeitos os táxons. $\mathrm{O}$ estresse hídrico é um dos fatores mais limitantes de produtividade e distribuição geográfica das espécies vegetais (Costa et al., 2010).

No bioma Caatinga ocorrem elevada radiação solar e altas taxas de evapotranspiração, por volta de 2.000 $\mathrm{mm}$. Nesse contexto, o conhecimento de como esses processos desencadeiam eventos sobre a vegetação (germinação, crescimento, ciclagem de nutrientes, biota do solo, dentre outros) é de grande importância para a consolidação de estratégias de utilização racional dos recursos locais da região e ajudam a explicar a adaptabilidade e persistência de muitas espécies nesse ecossistema (Parente, 2009).

$\mathrm{O}$ estudo de ciclagem de nutrientes em ecossistemas naturais pode, em médio e longo prazo, fornecer subsídios para um melhor entendimento das relações existentes naquela área e/ou região. De posse de informações relativas à deposição, acúmulo e decomposição da serapilheira é possível definir estratégias para o manejo sustentável de determinado ecossistema, a exemplo da Caatinga (Ferreira, 2011).

$\mathrm{Na}$ pesquisa sobre produção de serapilheira, destaca-se a importância desse material na conservação e manutenção natural dos ecossistemas florestais. Uma vez que, além dos processos naturais de ciclagem, esse material abriga em seu conteúdo grande quantidade de sementes em estado de dormência. Este estudo pode ser utilizado como um dos subsídios básicos para o manejo florestal, bem como para a detecção de distúrbios de origens natural e antrópica (Andrade et al., 2008).

Desta forma, o objetivo do estudo foi obter informações sobre o aporte do material formador da serapilheira, sua composição química e velocidade de decomposição em uma área de Caatinga no município de Santa Luz, PI.

\section{MATERIAL E MÉTODOS}

\subsection{Meio físico}

A área de realização do estudo localiza-se no município de Santa Luz, PI, latitude $8^{\circ} 57^{\prime} 14^{\prime \prime}$ S e longitude $44^{\circ} 07^{\prime} \mathrm{O}$ e situada a $345 \mathrm{~m}$ de altitude. O clima da região é classificado como Aw, tropical semiárido quente caracterizado por duas estações bem definidas, verão chuvoso e inverno seco, com duração de seis meses cada. A temperatura média é de $28^{\circ} \mathrm{Ce}$ precipitação anual, de $500 \mathrm{~mm}$ a $700 \mathrm{~mm}$.

Para o estudo foi selecionada uma área de Caatinga arbórea-arbustiva bem preservada, em uma área de 3 mil ha. O solo sob essa formação foi classificado como Neossolo Litólico.

\subsection{Aporte de serapilheira e quantificação de $N, P$ e K}

O aporte de serapilheira foi avaliado pelo uso de 10 coletores de formato circular com $0,15 \mathrm{~m}^{2}$ de área (perímetro igual a 1,40 m), dispostos acima da superfície do solo numa área delimitada de um hectare. Os coletores foram distribuídos aleatoriamente dentro de cada área, sendo presos ao caule de quatro árvores equidistantes com arame galvanizado a aproximadamente um metro de altura do solo. Os coletores foram instalados no mês de novembro de 2010 e as coletas foram realizadas mensalmente até novembro de 2011.

A produção de serapilheira foi estimada segundo Lopes et al. (2002), partindo-se da seguinte fórmula (Equação 1):

$\mathrm{PAS}=(\Sigma \mathrm{PS} \times 10.000) / \mathrm{Ac}$ 
em que: PAS = Produção média anual de serapilheira $\left(\mathrm{kg} \mathrm{ha}^{-1} \mathrm{ano}^{-1}\right)$; PS = Produção média mensal de serapilheira $\left(\mathrm{Mg} \mathrm{ha}^{-1} \mathrm{mês}^{-1}\right) ; \mathrm{Ac}=$ Área do coletor $\left(\mathrm{m}^{2}\right)$.

Posteriormente o material coletado foi seco em estufa a $65^{\circ} \mathrm{C}$, sendo separado em frações (folhas, galhos $<2 \mathrm{~cm}$ diâmetro, sementes, flores, casca e outros, correspondendo a fragmentos $<2 \mathrm{~mm}$ de diâmetro não identificáveis) e pesado. Uma subamostra do material foi moída em moinho tipo Willey para a realização da análise química, feita por meio de digestão sulfúrica (Tedesco et al., 1985), sendo determinados nitrogênio (N), pelo método de destilação de arraste a vapor (Bremner, 1960), fósforo (P), por colorimetria, e potássio (K), por fotometria de chama. Todos os resultados foram transformados para conteúdo de nutrientes. Também foram determinados no material vegetal os teores de polifenóis, lignina, tanino e celulose, segundo Abreu \& Albuquerque (1996).

Foi coletada a precipitação mensal na estação meteorológica do Inmet, localizada a $10 \mathrm{~km}$ da área de estudo, no campus da Universidade Federal do Piauí. Posteriormente foi realizada uma correlação de Pearson ao nível de $1 \%$ de significância.

Os dados referentes à serapilheira foram submetidos à análise estatística pelo teste de Tukey ao nível de significância de 5\%.

\subsection{Decomposição da serapilheira}

A avaliação da taxa de decomposição foi feita pela análise de perda de massa utilizando-se litter bags. Os litter bags consistiram em sacolas de polivinil com malha de $4 \mathrm{~mm}$ e dimensões de $25 \times 25 \mathrm{~cm}$ e $1,5 \mathrm{~cm}$ de altura. Em cada litter bag foram adicionadas 10 gramas de folhas do material aportado nos coletores cônicos, após esse ter sido seco em estufa a $65{ }^{\circ} \mathrm{C}$ até peso constante. Os litter bags foram distribuídos aleatoriamente na superfície do piso florestal, simulando a queda natural do material formador da serapilheira.

Os litter bags foram instalados no início do mês de agosto e coletados aos 30, 60, 90 e 120 dias. Após a coleta, os litter bags foram secos em estufa de circulação de ar forçada $\left(65 \pm 5^{\circ} \mathrm{C}\right)$ até atingirem peso constante. Em seguida, foram levados ao laboratório, onde o seu conteúdo foi examinado para retirada de partículas de solo, sendo sua massa posteriormente pesada em balança analítica de precisão. A taxa de decomposição da serapilheira foi quantificada mediante avaliações de medidas da perda de massa, com a seguinte fórmula (Equação 2):

Massa remanescente $(\%)=$

(massa final/massa inicial) x 100

Após o cálculo da massa remanescente ao longo do período, a constante de decomposição $\mathrm{k}$ foi calculada, segundo Thomas \& Asakawa (1993), conforme o modelo exponencial (Equação 3):

$\mathrm{Xt}=\mathrm{Xo} \cdot e^{-\mathrm{kt}}$

em que: $\mathrm{Xt}=$ Peso do material seco remanescente após $\mathrm{t}$ dias; $\mathrm{Xo}=$ Peso do material seco colocado nos sacos no tempo zero $(t=0)$.

Já o tempo de meia vida $\left(\mathrm{t}^{1 / 2}\right)$ foi calculado segundo Rezende et al. (1997), pela Equação 4:

$\mathrm{t}^{1 / 2}=\ln (2) / \mathrm{K}$

em que: $\mathrm{K}=$ Constante de decomposição calculada pela fórmula anterior $\left(\mathrm{Xt}=\mathrm{Xo} \cdot e^{-\mathrm{kt}}\right)$.

\section{RESULTADOS E DISCUSSÃO}

\subsection{Aporte de serapilheira e quantificação de $N, P$ e K}

A produção anual estimada de serapilheira foi de $8,44 \mathrm{Mg} \mathrm{ha}^{-1} \mathrm{ano}^{-1}$, sua deposição mensal e a relação com a precipitação é apresentada na Figura 1.

Os valores de produção mensal de serapilheira foram superiores aos do estudo de Costa et al. (2010), que ao avaliarem a produção de serapilheira em uma área preservada do bioma Caatinga no Rio Grande do Norte, observaram que em média a produção de serapilheira nesse bioma está em torno de 0,100 a 0,717 $\mathrm{Mg} \mathrm{ha}^{-1} \mathrm{mês}^{-1}$.

Amorim et al. (2008), avaliando deposição de serapilheira em área de Caatinga hiperxerófila preservada no município de Petrolina, PE, obtiveram valor médio de serapilheira total de 7,04 $\mathrm{Mg} \mathrm{ha}^{-1}$ ano $^{-1}$, semelhante ao observado neste estudo, devido às características edafoclimáticas e vegetacionais similares. Santos et al. (2011), avaliando a deposição de serapilheira em uma Caatinga antropizada e em processo de regeneração em Caruarú, PE, observaram uma deposição anual de 9,15 $\mathrm{Mg} \mathrm{ha}^{-1}$ ano $^{-1}$.

Quanto à deposição mensal de serapilheira verificouse que o mês de maior produção foi agosto $(1,87 \mathrm{Mg}$ 


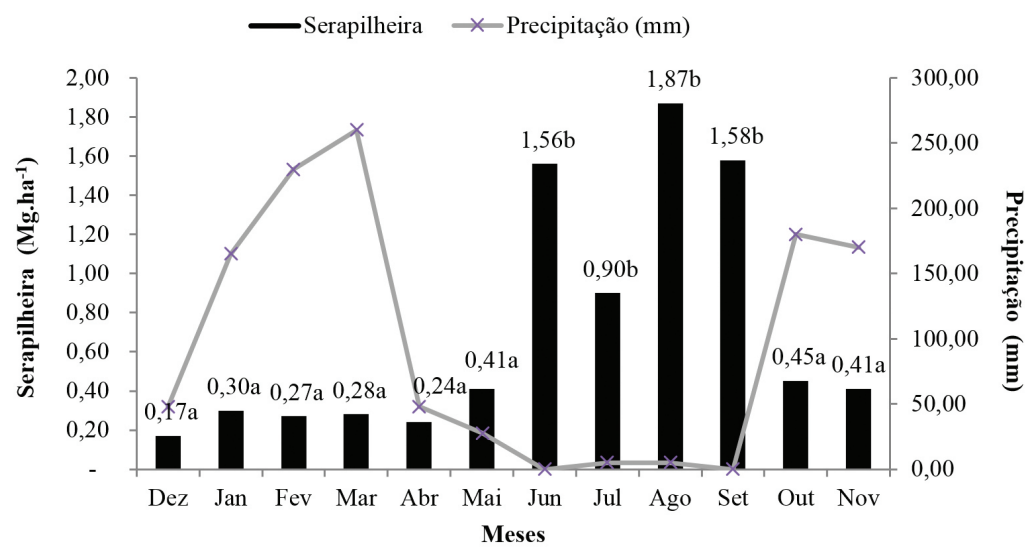

Figura 1. Precipitação e deposição da serapilheira mensal em área de Caatinga no município de Santa Luz, PI; letras iguais não diferem pelo teste Tukey ao nível 5\%.

Figure 1. Precipitation and deposition of litter in Caatinga area monthly in the municipality of Santa Luz, PI. Lyrics equal do not differ by the test of Tukey at $5 \%$.

$\left.\mathrm{ha}^{-1}\right)$ e o de menor, dezembro $\left(0,17 \mathrm{Mg} \mathrm{ha}^{-1}\right)$, sendo observada diferença estatística (Figura 1). Sanches et al. (2009) observaram produção média mensal de serapilheira variando de 0,05 a 1,53 $\mathrm{Mg} \mathrm{ha}^{-1} \mathrm{mês}^{-1} \mathrm{em}$ floresta de transição Amazônica e Cerrado no estado de Mato Grosso.

$\mathrm{Na}$ área de Caatinga foi observada uma correlação significativa ao nível de $1 \%\left(r^{2}=0,62\right)$ entre a deposição de serapilheira e a precipitação pluviométrica ao longo do período estudado, demonstrando que a precipitação influencia de forma significativa na deposição do material decíduo nessa área (Figura 1). Lopes et al. (2009) observaram uma correlação entre o total de chuva durante o período de estudo e a deposição da serapilheira em um trecho de Caatinga no município de Iguatu, $\mathrm{CE}$.

Entre os meses de junho a setembro ocorreram as maiores deposições de serapilheira, de forma que esse é o período de menor precipitação, no qual as espécies florestais perdem as folhas para reduzir a evapotranspiração (Figura 1). Esse comportamento está relacionado com a resposta da vegetação ao agravamento do estresse hídrico, sendo a queda de folhas medida preventiva à alta perda de água por transpiração, característica fenológica utilizada pelas espécies da Caatinga (Andrade et al., 2008).

Na Figura 2 é apresentado o total anual para os aportes de nitrogênio, fósforo e potássio. Nota-se que o nitrogênio foi o elemento de maior aporte, com $93,07 \mathrm{~kg} \mathrm{ha}^{-1}$ ano $^{-1}$, apresentando seus aportes mensais alta correlação com a serapilheira produzida $(0,78 ; \mathrm{p}<0,01)$. Pereira et al. (2012), avaliando a deposição de serapilheira em uma Restinga no Rio de Janeiro, observaram um aporte de nitrogênio de $71,90 \mathrm{~kg} \mathrm{ha}^{-1}$ ano $^{-1}$.

Para o fósforo $(\mathrm{P})$, verificam-se (Figura 2) valores totais anuais de aporte de $0,37 \mathrm{~kg} \mathrm{ha}^{-1}$ ano $^{-1}$, porém apresentando uma correlação menor com os aportes mensais de serapilheira $(0,20 ; \mathrm{p}<0,01)$. Os valores de fósforo podem ser considerados baixos quando comparados aos de outros ecossistemas; Gomes et al. (2010) observaram um aporte de fósforo de $3,05 \mathrm{~kg}$ $\mathrm{ha}^{-1}$ ano $^{-1}$ para fragmentos florestais de Mata Atlântica com diferentes tamanhos no município de Teresópolis, no estado do Rio de Janeiro. O potássio (K) foi o segundo elemento devolvido em maior quantidade, apresentando aportes anuais em torno de $2,08 \mathrm{~kg}$ $\mathrm{ha}^{-1} \mathrm{ano}^{-1}$, apresentando uma boa correlação com os aportes mensais $(0,60 ; \mathrm{p}<0,01)$. Caldeira et al. (2013) observaram aporte de $3,47 \mathrm{~kg} \mathrm{ha}^{-1} \mathrm{ano}^{-1}$ de K em um trecho de Mata Atlântica bem preservada no município de Alegre, ES.

$\mathrm{Na}$ distribuição percentual das frações que compõem a serapilheira, as folhas apresentaram entre os componentes a maior participação (76,55\%). A fração galhos foi a que apresentou o segundo maior percentual $(11,13 \%)$, porcentagem essa inferior às encontradas por Gonçalves (2008) e Fior et al. (2010) em Florestas Estacionais Semideciduais, que foram, respectivamente, de $15,45 \%$ e de $16 \%$ a $19 \%$. A fração 


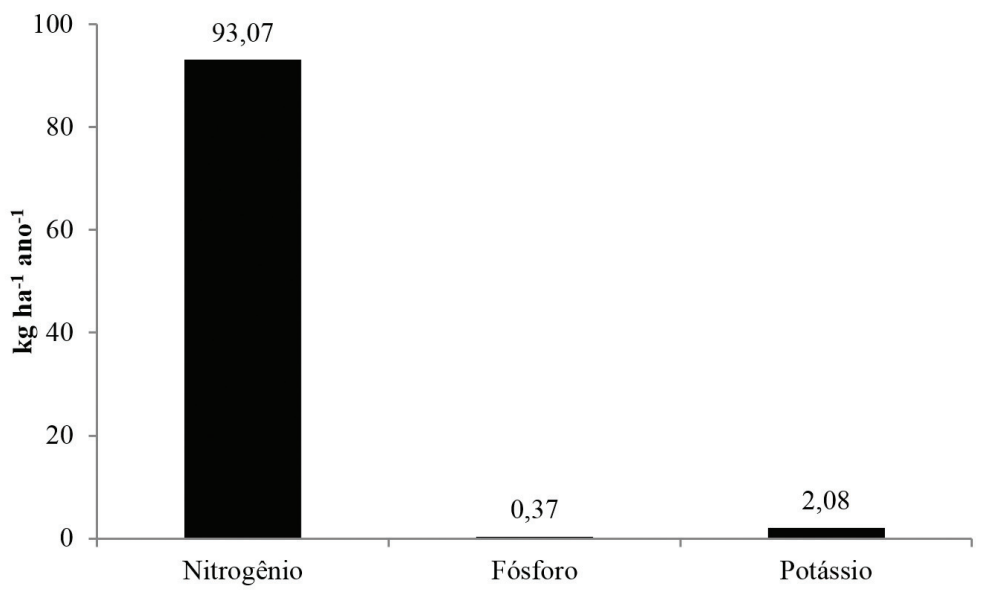

Figura 2. Aporte anual de nitrogênio, fósforo e potássio em área de Caatinga no município de Santa Luz, PI.

Figure 2. Annual Contribution of nitrogen, phosphorus and potassium in Caatinga area in the municipality of Santa Luz, PI.

casca corresponde a 4,45\%, a fração sementes, a 3,66\% e as flores, a 1,93\%; o restante que se refere a fração miscelânea e corresponde a 2,28\% do total (Figura 3).

Em trabalho avaliando a contribuição de folhas na formação da serapilheira no norte do Mato Grosso, em área de transição Amazônia-Cerrado, Silva et al. (2009) observaram maior produção de serapilheira no período seco, com picos também nos meses de agosto e setembro, mas com $70 \%$ de folhas, $12 \%$ de galhos, $12 \%$ de frutos e $6 \%$ de Flores. Gomes et al. (2010) observaram, em média, 69,3\% de folhas do total do material aportado, seguida pela fração galhos variando entre $11,9 \%$ e 16,3\%, valores semelhantes ao deste estudo (Figura 3).

\subsection{Decomposição da serapilheira}

A constante de decomposição (K) é uma estimava da proporção de decomposição, em um ano, da camada de serapilheira acumulada sobre o solo. Essa constante de decomposição (k) foi estimada em 0,20 g g-1 dia-1 $\left(\mathrm{P}<0,01 ; \mathrm{r}^{2}=0,91\right)$, sendo essa referente à velocidade de decomposição. O tempo de meia-vida $\left(\mathrm{t}^{1 / 2}\right)$ estimado para o período esteve em torno de 493 dias (Figura 4).

$\mathrm{O}$ valor $\mathrm{K}$ observado neste estudo pode ser considerado baixo quando comparado a outros estudos de decomposição na Caatinga. Lopes et al. (2009), trabalhando com a decomposição da serapilheira em Caatinga no estado do Ceará, observaram um valor K de 0,71. Santana (2005), em uma Caatinga no Rio Grande do Norte, obteve um valor de K de 0,33, enquanto que Souto (2006), no sertão paraibano, encontrou um valor de $\mathrm{K}$ igual a 1,25.

A equação que descreve a velocidade de decomposição da área de Caatinga é o modelo matemático y $=130,01_{e}^{-0,223 x}$, com índice de regressão de 0,915, apresentando significância a 1\%, demonstrando que a decomposição ocorre de forma exponencial e significativa. Observa-se que a velocidade de decomposição é lenta, sendo que aos 120 dias mais de 50\% do material remanescente ainda não foi decomposto (Figura 4).

A baixa decomposição observada neste estudo pode ser explicada pelos altos teores de lignina e tanino contidos na serapilheira (Tabela 1). A lignina torna a serapilheira mais resistente à decomposição química promovida por fungos e bactérias, e o tanino torna a serapilheira pouco palatável para certos grupos da meso e macrofauna edáfica (Correia \& Andrade, 1999). Outro fator que influencia na decomposição lenta é a baixa umidade do solo da área de Caatinga, que reduz a atividade dos organismos decompositores da serapilheira (Santana \& Souto, 2011).

Na Tabela 1 são apresentados os valores de lignina e tanino da serapilheira da área em estudo. $\mathrm{O}$ valor de lignina $\left(497,93 \mathrm{~g} \mathrm{~kg}^{-1}\right)$ pode ser considerado alto, conforme Pereira et al. (2012), que observaram valores de 465,80 $\mathrm{g} \mathrm{kg}^{-1}$ em vegetação de Restinga. Os valores foram considerados altos em função das condições ambientais onde as espécies, como estratégia para a redução das perdas por transpiração, apresentam as folhas com textura coriácea. 


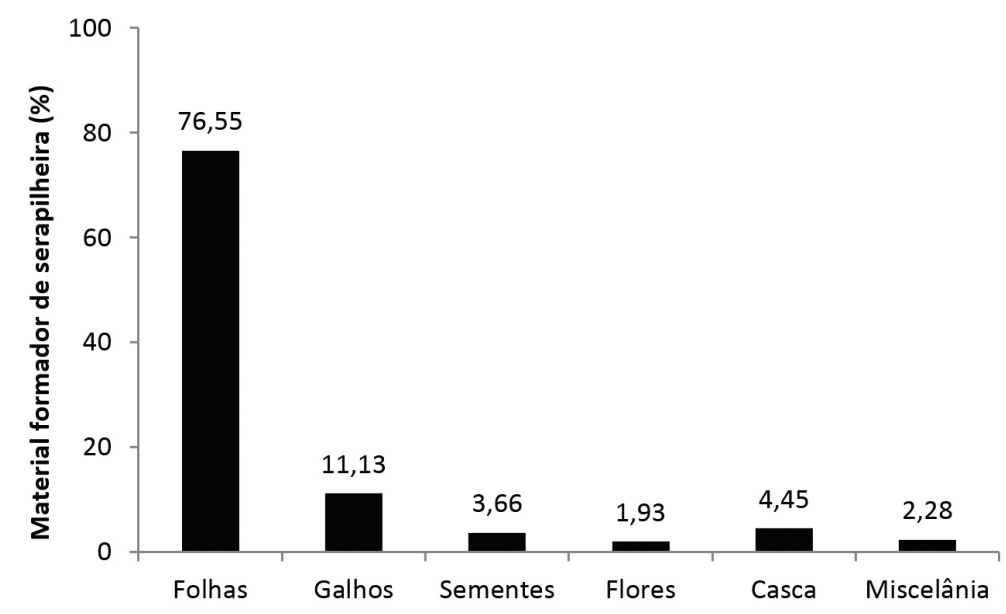

Figura 3. Frações do material decíduo na área de Caatinga durante o período de estudo.

Figure 3. Deciduous material fractions in the Caatinga area during the period of study.

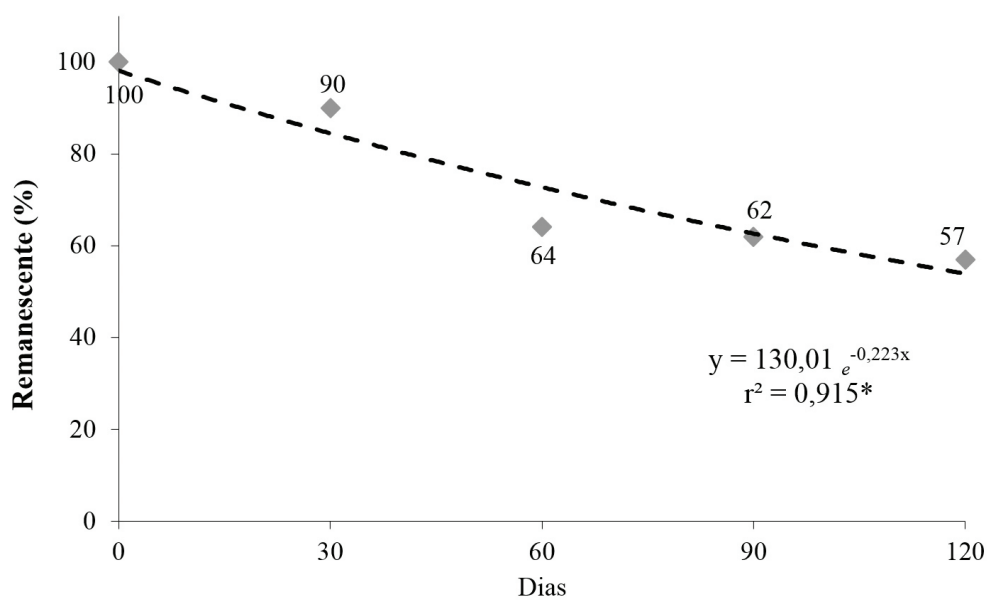

Figura 4. Curva de decomposição da área de Caatinga. ${ }^{\star}$ Valores significativos a 5\%.

Figure 4. Decomposition curve of the Caatinga area. ${ }^{\star}$ Values significant at $5 \%$.

Tabela 1. Teores de lignina e tanino na serapilheira foliar da área de Caatinga no município de Santa Luz, PI.

Table 1. Lignin and tannin levels in foliar litterfall of the Caatinga area in the municipality of Santa Luz, PI.

\begin{tabular}{cc} 
Componente & $\left(\mathbf{g ~ k g}^{-1}\right)$ \\
\hline Lignina & 497,93 \\
Tanino & 263,00 \\
\hline
\end{tabular}

Em ecossistemas florestais com baixa precipitação e elevada evapotranspiração como a Caatinga existe uma maior lignificação das folhas, que visa diminuir a condutância estomática e, consequentemente, minimizar as perdas hídricas por transpiração com a presença de muitas folhas coriáceas (Santana, 2005).

Os valores de tanino observados neste estudo podem ser considerados altos quando comparados aos observados por Pereira et al. (2012) em uma Restinga no Rio de Janeiro, no período de janeiro a dezembro de 2002, que registraram valores de tanino na serapilheira em torno de $48,60 \mathrm{~g} \mathrm{~kg}^{-1}$.

Quanto às transformações ocorrentes no processo de decomposição da matéria orgânica, Waksman (1952) sugere duas fases principais: a primeira, de 1 a 2 meses, na qual ocorre a perda de compostos solúveis, e a segunda, de ataque aos compostos estruturais que podem perdurar durante anos, dependendo da qualidade do material. Portanto, verifica-se que com o passar do tempo tem-se a princípio uma rápida perda da maioria dos hidrossolúveis, uma diminuição lenta de compostos hidrossolúveis fenólicos (tanino, por exemplo) e hemiceluloses e um aumento relativo do conteúdo de lignina. 


\section{CONCLUSÕES}

A redução da precipitação levou ao aumento da deposição de serapilheira, principalmente da fração folhas. O nitrogênio apresentou o maior aporte dentre os nutrientes.

Os elevados teores de lignina e tanino na serapilheira da Caatinga reduzem a velocidade da decomposição da serapilheira.

\section{STATUS DA SUBMISSÃO}

Recebido: 9 out., 2013

Aceito: 4 nov., 2014

\section{AUTOR(ES) PARA CORRESPONDÊNCIA}

\section{Milton Marques Fernandes}

Departamento de Ciências Florestais,

Universidade Federal de Sergipe, CEP 49075-250,

Aracaju, SE, Brasil

e-mail: miltonmf@gmail.com

\section{REFERENNCIAS}

Abreu HS, Albuquerque CEC. Receituário químico. Rio de Janeiro: Imprensa Universitária; 1996.

Amorim LB, Salcedo IH, Galindo ICL, Silva VF. Estoque de serapilheira em área de caatinga preservada. In: Anais da Reunião Brasileira de Manejo e Conservação do Solo e da Água; 2008; Rio de Janeiro. Rio de Janeiro: UFRRJ; 2008. p. 28-32.

Andrade RL, Souto JS, Souto PC, Bezerra DM. Deposição de serrapilheira em área de Caatinga na RPPN "Fazenda Tamanduá", Santa Terezinha - PB. Caatinga 2008; 21(2): 223-230.

Bremner JM. Nitrogen-total: determination of nitrogen in soil by Kjedahl method. The Journal of Agricultural Science 1960; 55(1): 11-33. http://dx.doi.org/10.1017/ S0021859600021572.

Caldeira MVW, Silva RD, Kunz SH, Zorzanelli JPF, Castro KC, Godinho TO. Biomassa e nutrientes da serapilheira em diferentes coberturas florestais. Comunicata Scientiae 2013; 4(2): 111-119.

Correia MEF, Andrade AG. Formação de serapilheira e ciclagem de nutrientes. In: Santos GA, Camargo FAO, editores. Fundamentos da matéria orgânica do solo: ecossistemas tropicais e subtropicais. Porto Alegre: Gênesis; 1999.
Costa CCA, Camacho RGV, Macedo ID, Silva PCM. Análise comparativa da produção de serapilheira em fragmentos arbóreos e arbustivos em área de caatinga na Flona de Açu - RN. Árvore 2010; 34(2): 259-265. http:// dx.doi.org/10.1590/S0100-67622010000200008.

Ferreira CD. Deposição, acúmulo e decomposição de serapilheira em área de caatinga preservada [monografia]. Campina Grande: Centro de Saúde e Tecnologia Rural, Universidade Federal da Paraíba; 2011.

Fior RC, Soares MR, Casagrande JC, Guerra F, Moraes MIM. Produção e decomposição de serapilheira de mata ciliar em dois estádios de sucessão ecológica. In: Anais do FERTBIO; 2010; Guarapari, ES.

Gomes JM, Pereira MG, Piña-Rodrigues FCM, Pereira GHA, Gondim FR, Silva EMR. Aporte de serapilheira e de nutrientes em fragmentos florestais da Mata Atlântica. Revista Brasileira Ciências Agrária 2010; 5(3): 383-391. http://dx.doi.org/10.5039/agraria.v5i3a552.

Gonçalves MAM. Avaliação da serapilheira em fragmento de floresta atlântica no sul do estado do Espírito Santo [dissertação]. Alegre: Centro de Ciências Agrárias, Universidade Federal do Espírito Santo; 2008.

Lopes MIS, Domingos M, Struffaldi-de-Vuono Y. Ciclagem de nutrientes minerais. In: Syslvestre LS, Rosa MMT, editores. Manual metodológico para estudos botânicos na Mata Atlântica. Seropédica: Universidade Federal Rural do Rio de Janeiro; 2002.

Lopes JFB, Andrade EM, Lobato FAO, Palácios HAQ, Arraes FDD. Deposição e decomposição de serapilheira em área da Caatinga. Revista Agro@mbiente On-line 2009; 3(2): 72-79.

Parente HN. Avaliação da vegetação e do solo em áreas de caatinga sob pastejo caprino no cariri da Paraíba [tese]. Areia: Centro de Ciências Agrárias, Universidade Federal da Paraíba; 2009.

Pereira MG, Silva NA, Paula RR, Menezes LFT. Aporte e decomposição de serapilheira em floresta periodicamente inundável na restinga da Marambaia, RJ. Ciência Floresta2012; 22(1): 59-67.

Pinto SIC, Martins SV, Barros NF, Dias HCT. Produção de serapilheira em dois estádios sucessionais de floresta estacional semidecidual na Reserva Mata do Paraíso, em Viçosa, MG. Árvore 2008; 32(3): 545-556. http://dx.doi. org/10.1590/S0100-67622008000300015.

Rezende JP, Garcia QS, Scotti MR, Leitão MM. Decomposição de folhas de Dalbergia nigra e de Eucalyptus grandis incubadas em terra de mata e de eucaliptal. In: Anais do $3^{\circ}$ Simpósio Nacional de Recuperação de Áreas Degradadas: do Substrato ao Solo; 1997; Ouro Preto. Ouro Preto: SOBRADE;UFV;DEF; 1997. p. 136-143.

Sanches L, Valentini CMA, Biudes MS, Nogueira JS. Dinâmica sazonal da produção e decomposição de serrapilheira em 
floresta tropical de transição. Revista Brasileira Engenharia Agrícola Ambiental 2009; 13(2): 183-189.

Santana JAS. Estrutura fitossociológica, produção de serapilheira e ciclagem de nutrientes em uma área de Caatinga no Seridó do Rio Grande do Norte [tese]. Areia: Universidade Federal da Paraíba; 2005.

Santana JAS, Souto JS. Produção de serapilheira na Caatinga da região semiárida do Rio Grande do Norte, Brasil. IDESIA 2011; 29(2): 87-94. http://dx.doi.org/10.4067/ S0718-34292011000200011.

Santos PS, Souza JT, Santos JMFF, Santos DM, Araujo EL. Diferenças sazonais no aporte de serrapilheira em uma área de Caatinga em Pernambuco. Revista Caatinga 2011; 24(4): 94-101.

Silva CJ, Lobo FA, Bleich ME, Sanches L. Contribuição de folhas na formação da serrapilheira e no retorno de nutrientes em floresta de transição no norte de Mato Grosso. Acta Amazonica 2009; 39(3): 591-600. http:// dx.doi.org/10.1590/S0044-59672009000300014.

Souto PC. Acumulação e decomposição da serapilheira e distribuição de organismos edáficos em área de Caatinga na Paraíba [tese]. Areia: Universidade Federal da Paraíba; 2006.

Tedesco MJ, Volkweiss SJ, Bohnen H. Análises de solo, plantas e outros materiais. 5. ed. Porto Alegre: UFRRGS; $1985.50 \mathrm{p}$.

Thomas RJ, Asakawa NM. Decomposition of leaf litter from tropical forage grasses and legumes. Soil Biology \& Biochemistry 1993; 25(10): 1351-1361. http://dx.doi. org/10.1016/0038-0717(93)90050-L.

Waksman S. Principles of soil microbiology. 2nd ed. Baltimore: Wilians \& Wilkings Company; 1952. 894 p. 